\title{
Probabilistic modeling of hydraulic conditions of tree pipeline networks under conventional boundary conditions*
}

\author{
Olga V. Vanteeva ${ }^{1, \dagger}$ \\ ${ }^{1}$ Melentiev Energy Systems Institute of Siberian Branch of the Russian Academy of Sciences (ESI SB \\ RAS), Pipeline Energy Systems Department, 130, Lermontov str., Irkutsk, Russia, 664033
}

\begin{abstract}
The paper is concerned with the probabilistic modeling of pipeline network hydraulic conditions that occur due to random impacts of the external environment (load of consumers, pressures at sources). The research is focused on a network with a tree configuration under conventional boundary conditions (for pressure at a tree root and flow rates at the other nodes) that are specified probabilistically. We propose a topological method for a probabilistic analysis of such network operation. This method makes it possible to determine mathematical means, variances and covariances of state variables (pressures at nodes, flow rates and pressure losses in branches) by final formulas when traversing the entire tree without laborious matrix operations or calculations of systems of equations. A numerical case study is used to exemplify the efficiency of the proposed approach, adequacy of the obtained results and high speed compared to other, more general methods similar to Monte-Carlo methods.
\end{abstract}

\section{Introduction}

Flow distribution problems are primary in an analysis and substantiation of operating conditions of pipeline systems of various types and purposes for their design, operation and dispatch control. Traditionally, these problems are solved with deterministic mathematical models and methods, which however do not allow the assessment of the extent to which the pipeline system operation affected by a set of random factors is uncertain. This explains the relevance of the probabilistic flow distribution problem statements, which imply obtaining calculation results in the form allowing probabilistic interpretation based on the information on the so called boundary conditions in a probabilistic form. Solving such problems with traditional methods, for example with Monte Carlo method [1], is associated with excessive computational efforts even for the pipeline systems of a medium size.

\section{Analysis of literature and problem statement}

To date, Russia and other countries have carried out many studies dealing with the tasks

\footnotetext{
*The research was carried out within the project III.17.4.3 of the Fundamental research program of SB RAS (AAAA-A17-117030310437-4)

† Corresponding author: vanteeva@isem.irk.ru
} 
and methods for calculation of steady-state flow distribution in pipeline systems of different types and purposes.

The experience of flow distribution modeling in the papers by E. Todini [2-4], O. Giustolisi [5,6], is characterized mainly by their focus on deterministic, steady-state and quasidynamic flow distribution. In Russia, the flow distribution problems similar in statement were solved already in the 1930s by Andriyashev M.M. [7], and Lobachev V.G. [8], and generalized in the studies by A.P.Merenkov and V.Y.Khasilev [9].

The issues of stochastic flow distribution and optimization of parameters under uncertainty in water supply systems (in their design) are considered in the studies by S.N.Karambirov [10, 11]. In these studies, the statistical models (time series, autocorrelation functions) are used to take into account the stochastic nature of water consumption. The authors of $[12,13]$ put an emphasis on probabilistic problems considered in the analysis of pipeline system operating conditions, given pumps and reservoirs. These problems are mainly solved by Monte Carlo method which is also widely used in the analysis of pipeline system reliability.

The authors of [14-18] propose an approach to the development of analytic probabilistic models of steady-state hydraulic conditions. This approach, for example, can be applied to the water and heat supply systems under stochastic water consumption. The general and stage-by-stage methods presented in [14-17] are characterized by their universality, satisfactory accuracy and low computational efforts compared to the classical Monte Carlo method. They can be used to determine all probabilistic characteristics of steady-state conditions in pipeline systems of random configuration and any set of boundary conditions specified in a probabilistic form. The methods are aimed at obtaining mathematical means for state variables and calculating a covariance matrix of these variables based on a specified covariance matrix of boundary conditions and a derivative matrix of flow distribution model at the point of mathematical mean of boundary conditions, and involve matrix operations including inverse of a derivative matrix [14-18].

\section{Object, goal and objectives of studies}

An object of the research discussed in the paper is a tree pipeline network. The goal is to develop an effective method and an algorithm for calculation of statistic characteristics (mathematical means, variances, and covariances) of hydraulic state variables (pressures, flow rates) that occurred as a result of random impact of the external environment and that are specified probabilistically for pressure at one node (a tree root) and flow rates at the other nodes.

To achieve this goal we set the following objectives for the research: 1) to obtain final formulas for calculation of statistic characteristics of state variables; 2) to develop a topological method ( and an algorithm for calculation), that identify the sequence in which the obtained formulas should be applied; 3 ) to numerically check the operability of the topological method and accuracy of the obtained solution in comparison with the other methods (Monte Carlo and general methods).

\section{The main principles of the topological method}

We will introduce the notion of a tier of nodes with index $k$, which represents a set of nodes $J_{k}$, such that $\left|R_{j}\right|=k$, where $R_{j}$ - a set of branches belonging to the path from a tree root to node $j$. Thus, the $k$-th tier contains all nodes of the scheme that are connected with root $k$ by branches. Let us put the direction of branches of the tree scheme in correspondence with the direction of flows. Assign the first numbers (indices) to the nodes of the last tier, the 
next numbers - to the tier before last, and so on. Only one flow goes into each node, therefore the branches can always be numbered so that the branch index will coincide with the index of a final node, i.e. $i=\mathrm{f}_{i}=j$. Let us introduce a set of branches $I_{j}$, incident to node $j$, that can now be represented by $I_{j}=j \cup I_{j}^{-}$, where $I_{j}^{-}$is a set of branches going from node $j$.

By virtue of the mentioned principle of node and branch numbering, we obtain:

1) $\mathrm{o}_{i}>\mathrm{f}_{i}, i=\overline{1, n}$, where $\mathrm{o}_{i}, \mathrm{f}_{i}$ are the indices of initial and final nodes of the $i$-th branch;

2) node with an external source (tree root) will have index $m$;

3) $J_{0}=\{m\}, J_{k+1}=\bigcup_{j \in J_{k}} I_{j}^{-}, k=\overline{1, K-1}$;

4) the number of tiers in the scheme will be determined as $K=\left|R_{1}\right|+1$;

5) $j>l$ for all $j \in J_{k}$ and $l \in J_{k+1}, k=\overline{0, K-1}$, therefore, the enumeration of nodes $j=1, \ldots, m$ means a successive traversing through the tiers in the direction of $k=K-1, \ldots, 0$ and on the contrary, enumeration of nodes $j=m, \ldots, 1$ implies traversing in the direction of $k=0, \ldots, K-1$. Figure 1 demonstrates an example of introduced numbering.

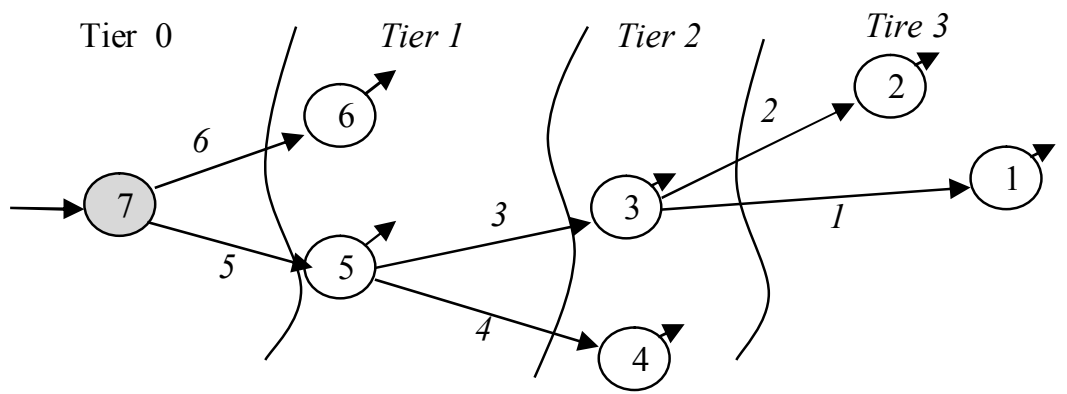

Fig.1. An example of a tree scheme numbered for calcualtion by the topological method.

The topological method implies successively traversing the nodes of the calculation scheme by tiers, starting with the nodes for which the boundary conditions are specified with relatively simple algebraic relationships in each step to determine the unknown values of mathematical means, variances and covariances of state variables.

From now on we assume that the set of boundary conditions (vector $G$ ) is conventional $G=\left(Q_{1}, Q_{2}, \ldots, Q_{m-1}, P_{m}\right)^{\mathrm{T}}$, where $Q_{j}, P_{j}$ are flow rate and pressure at the $j$-th node. Boundary conditions are specified by their mathematical means $\bar{G}$ and covariance matrix $C_{G}$. Without loss of generality, for simplicity we will also consider all boundary conditions to be mutually uncorrelated, when $\operatorname{cov}\left(G_{j}, G_{l}\right)=0$ for $j \neq l$ and $\operatorname{cov}\left(G_{j}, G_{l}\right)=\sigma_{j}^{2}$ for $j=l, j, l=\overline{1, m}$, i.e. $C_{G}=\operatorname{diag}\left(\sigma_{Q 1}^{2}, \sigma_{Q 2}^{2}, \ldots, \sigma_{Q m-1}^{2}, \sigma_{P m}^{2}\right)$ is a diagonal matrix of order $\operatorname{dim}(G)=m$.

\section{Calculation of mathematical means of state variables}

Let us explain the principle of traversing the nodes of the scheme on the example of a 
calculation of mathematical means of state variables.

Linearly independent equations of the first Kirchhoff law [9] will be presented in an index form: $x_{j}-\sum_{i \in I_{j}^{-}} x_{i}=Q_{j}, j=\overline{1, m-1}$, where $x_{i}$ is flow rate in the $\mathrm{i}$-th branch. The mathematical means of flow rates in the branches are calculated successively by the expression

$$
\bar{x}_{i}=\sum_{t \in I_{i}^{-}} \bar{x}_{t}+\bar{Q}_{i}, i=\overline{1, n},
$$

which takes into account the fact that $i>t \in I_{i}^{-}$. Therefore, by the time of calculating $\bar{x}_{i}$, all $\bar{x}_{t}, t \in I_{i}^{-}$are already known.

The pressure drop in branches $y_{i}=f_{i}\left(x_{i}\right), i=\overline{1, n}$, where $f_{i}\left(x_{i}\right)$ is function for hydraulic characteristics of the branches, therefore, $(1)$ can be used to calculate

$$
\bar{y}_{i} \approx f_{i}\left(\bar{x}_{i}\right), i=\overline{1, n} \text {. }
$$

The remaining linearly dependent equation of the first Kirchhoff law makes it possible to determine

$$
\bar{Q}_{m}=\sum_{i \in I_{m}^{-}} \bar{x}_{i} .
$$

The mathematical means of nodal pressures are calculated using an index analog of equations of the second Kirchhoff law in a nodal form $P_{\mathrm{o} i}-P_{\mathrm{f} i}=y_{i}$, hence

$$
\bar{P}_{j}=\bar{P}_{\mathrm{o} j}-\bar{y}_{j}, \quad j=\overline{n, 1} \text {. }
$$

The calculation starts for the nodes of tier $k=1$, when $\mathrm{H}_{j}=m$, and $\bar{P}_{m}$ is known. In calculation of $\bar{P}_{j}$ for $k>1$, the values of $\bar{P}_{\mathrm{o} j}$ are also known, because $\mathrm{o}_{j}>j$.

\section{Index-recurrent relationships for variances and covariances of state variables}

We will present the final relationships for the variances of state variables [18]:

1) for the variance of flow rates in branches

$$
\sigma_{x, i}^{2}=\sigma_{Q, i}^{2}+\sum_{t \in I_{i}^{-}} \sigma_{x, t}^{2}, i=\overline{1, n}
$$

2) for the variance of pressure drops in branches

$$
\sigma_{y, i}^{2}=\left(f_{x, i}^{\prime}\right)^{2} \sigma_{x, i}^{2}, i=\overline{1, n}
$$

where $f_{x, i}^{\prime}=\partial f_{i} / \partial x_{i}, i=\overline{1, n}$;

3) for the variance of flow rates in branches

$$
\sigma_{P, j}^{2}=\sigma_{P, \mathrm{H} j}^{2}+\sigma_{y, j}^{2}+2 f_{x, j}^{\prime} \sigma_{x, j}^{2} f_{\Sigma, \mathrm{H} j}, j=\overline{m-1,1} ;
$$

The value of $f_{\Sigma, i}$ is calculated recurrently -

$$
f_{\Sigma, m}=0, f_{\Sigma, j}=f_{\Sigma, \mathrm{H} j}+f_{x, j}^{\prime}, j=\overline{m-1,1} ;
$$

4) for the variance of the nodal flow rate at the source

$$
\sigma_{Q, m}^{2}=\sum_{t \in I_{m}^{-}} \sigma_{x, t}^{2} .
$$

\section{Topological algorithm}


Thus, the proposed "topological" method for probabilistic modeling of hydraulic conditions for a tree network can be reduced to the following computational scheme.

1. Forward pass. For each $i=\overline{1, n}$, calculate: $\bar{x}_{i}, \sigma_{x, i}^{2}$ with respect to (1), (5), and $\bar{y}_{i}, \sigma_{y, i}^{2}$ with respect to (2), (6). Determine $\bar{Q}_{m}, \sigma_{Q, m}^{2}$ with respect to (3), (9).

2. Backward pass. By assuming $f_{\Sigma, m}=0$, for each $j=\overline{m-1,1}$, determine $\bar{P}_{j}$ and $\sigma_{P, j}^{2}$ with respect to (4), (7), and $f_{\Sigma, j}$ - with respect to (8).

\section{Numerical case study}

Let us compare the probabilistic flow distribution characteristics for the scheme in Figure 1, that were calculated by different methods: Mote Carlo method, general method and topological method. The boundary conditions represent a set of mutually uncorrelated magnitudes $G=\left\{Q_{1}, \ldots, Q_{6}, P_{7}\right\}$ with numerical values of mathematical means $\bar{G}=\{50 ; 5 ; 5 ; 3 ; 8,06 ; 5 ; 7\}$ and mean square deviations $\{0,1 ; 0,86 ; 0,87 ; 0,3 ; 0,887 ; 0,5 ; 1,264\}$. The functions for hydraulic characteristics of branches have the form $f_{i}\left(x_{i}\right)=s_{i}\left|x_{i}\right| x_{i}, i=\overline{1, n}$, where $s_{i}$ - hydraulic resistance of the $i$-th branch. These resistances have the values $\{0,015 ; 0,0908 ; 0,0108 ; 0,015 ; 0,00646$; $0,00557\}$. Consequently, $\partial f_{i} / \partial x_{i}=2 s_{i}\left|x_{i}\right|, i=\overline{1, n}$.

Tables 1 and 2 contain calculated probabilistic characteristics for flow rates in the branches and nodal pressures by different methods. The characteristics of the nodal flow rate of the sources found by different methods are: $\bar{Q}_{7}=33,07, \sigma_{Q, 7}^{2}=2,046(\mathrm{MCM})$; $\bar{Q}_{7}=33,06, \sigma_{Q, 7}^{2}=2,054$ (GM and SSM).

Table 1. Results of probabilistic modeling of flow rates in branches

\begin{tabular}{|c|c|c|c|c|c|c|}
\hline \multirow{2}{*}{ Branch, $i$} & \multicolumn{2}{|c|}{ Monte Carlo method $(\mathrm{N}=5000)$} & \multicolumn{2}{c|}{ General method } & \multicolumn{2}{|c|}{ Topological method } \\
\cline { 2 - 7 } & $\bar{x}_{i}$ & $\sigma_{x, i}$ & $\bar{x}_{i}$ & $\sigma_{x, i}$ & $\bar{x}_{i}$ & $\sigma_{x, i}$ \\
\hline 1 & 4,99 & 0,86 & 5 & 0,86 & 5 & 0,86 \\
\hline 2 & 4,99 & 0,88 & 5 & 0,87 & 5 & 0,87 \\
\hline 3 & 12,99 & 1,27 & 13 & 1,26 & 13 & 1,26 \\
\hline 4 & 8,06 & 0,88 & 8,06 & 0,88 & 8,06 & 0,88 \\
\hline 5 & 26,04 & 1,61 & 26,06 & 1,62 & 26,06 & 1,62 \\
\hline 6 & 7,01 & 1,26 & 7 & 1,26 & 7 & 1,26 \\
\hline
\end{tabular}

Table 2. Results of probabilistic modeling of nodal pressures

\begin{tabular}{|c|c|c|c|c|c|c|}
\hline \multirow{2}{*}{ Node,$j$} & \multicolumn{2}{|c|}{ Monte Carlo method $(\mathrm{N}=5000)$} & \multicolumn{2}{c|}{ General method } & \multicolumn{2}{c|}{ Topological method } \\
\cline { 2 - 7 } & $\bar{P}_{i}$ & $\sigma_{P, j}$ & $\bar{P}_{i}$ & $\sigma_{P, j}$ & $\bar{P}_{i}$ & $\sigma_{P, j}$ \\
\hline 1 & 43,40 & 0,94 & 43,41 & 0,94 & 43,41 & 0,94 \\
\hline 2 & 41,51 & 1,49 & 41,51 & 1,48 & 41,51 & 1,48 \\
\hline 3 & 43,77 & 0,85 & 43,78 & 0,85 & 43,78 & 0,85 \\
\hline 4 & 44,62 & 0,69 & 44,63 & 0,69 & 44,63 & 0,69 \\
\hline 5 & 45,62 & 0,55 & 45,61 & 0,55 & 45,61 & 0,55 \\
\hline 6 & 49,72 & 0,14 & 49,72 & 0,14 & 49,72 & 0,14 \\
\hline 7 & 50,00 & 0,1 & 50,00 & 0,1 & 50,00 & 0,1 \\
\hline
\end{tabular}


As is seen, the results obtained by different methods virtually coincide. The calculation time with the topological method, however, is many times less than with general and Monte Carlo methods: Monte Carlo method (5000 realizations) - 206 s; General method - 1 s.; Topological method $-0.25 \mathrm{~s}$.

\section{Conclusions}

The probabilistic modeling of flow distribution in the pipeline system of a tree configuration has been studied. The final expressions for the mathematical means and variances of state variables are calculated recurrently. A new topological method and an algorithm are developed to calculate all statistic parameters of hydraulic conditions while traversing the tree. The proposed method enables calculations without laborious matrix operations or solving the systems of equations. The numerical cases are used to exemplify the adequacy of the results obtained with the proposed method and its greater computational efficiency compared to the other alternative methods.

\section{References}

1. I.M. Sobol. Numerical Monte Carlo methods (Science, Moscow, 1973) [in Russian].

2. Todini, E. Bollettinodegli Ingegneridella. Toscana 11 (1979)

3. Todini E., Pilati S. A gradient algorithm for the analysis of pipe networks. (L., John Wiley \& Sons, 1988)

4. Todini, E., Computing and Control for the water Industry (1999)

5. O. Giustolisi, Z. Kapelan, D. Savic, J. of water resources planning and management. 134, 6 (2008)

6. O. Giustolisi. Proc. of WEX Workshop, Marbella, Spain. 2008. (CD-ROM)

7. M.M. Andriyashev. A technique for calculation of water pipeline networks (M., Soviet Legislature, 1932)

8. V.G. Lobachev. Santekhnika. 2, (1934)

9. A.P. Merenkov and V.Ya. Khasilev, The Theory of Hydraulic Circuits (Science, Moscow, 1985)

10. S.N. Karambirov. Mathematical modeling of systems for supply and distribution of water under a great number of operating conditions and uncertainty (M., MGUP, 2004)

11. Karambirov S.N. Abstract of PhD Thesis (M., 2005)

12. H.A. Kretzmann, J.E. van Zyl and J. Haarhoff, Proceedings of the 2004 Water Institute of Southern Africa (WISA) (Biennial Conference 2 - 6 May 2004)

13. P. Cutore, A. Campisano, Z. Kapelan, C. Modica, D. Savic. Urban Water Journal, 5, 2 (2008)

14. N.N. Novitsky, O.V. Vanteeva. Saint Petersburg Polytechnic University Journal of Engineering Science and Technology, 1 (2008)

15. N.N. Novitsky, O.V. Vaneeva, Proceedings of the Russian Academy of Sciences. Power Engineering, 2 (2011)

16. O.V. Vanteeva. Abstract of PhD Thesis (Irkutsk ESI SB RAS, 2011)

17. N.N. Novitsky, O.V. Vanteyeva. Chaotic Modeling and Simulation (CMSIM), 1 (2014)

18. N.N. Novitsky, O.V. Vanteeva, Proceedings of the Russian Academy of Sciences. Power Engineering, 6 (2017) 